\title{
Superando la ansiedad para lograr el aprendizaje de una segunda lengua
}

Overcoming anxiety to achieve the learning of a second
language

Carlos Alberto Armendáriz Valles ${ }^{1}$

\section{Resumen}

El presente proyecto de intervención fue diseñado con base en la necesidad de ayudar a los alumnos a controlar la ansiedad que les produce el aprendizaje de una segunda lengua, se interviene educativamente con el fin de trabajar estrategias que les permitan lograr ser conscientes de la ansiedad que les genera las prácticas y ejercicios orales, escritos y auditivos en la asignatura de inglés; también se pretende que elaboren estrategias de autocontrol, facilitando el aprendizaje y manejo pertinente del idioma, así como maniobrar con la ansiedad negativa a fin de convertirla en positiva e impulsar el aprendizaje de la segunda lengua. El diagnóstico realizado revela que los alumnos reconocen la ansiedad y saben que es un mecanismo de defensa por la inseguridad que tienen al aprender una lengua extranjera, esto les permite controlar esta emoción dando como resultado un mejor aprendizaje del idioma. Las estrategias fueron un taller con siete actividades los cuales van encaminadas a crear un clima de confianza y reconocimiento de los procesos psicológicos que se viven al estar en un grupo y proporcionar seguridad al alumno, estimulando a los estudiantes para que enfrenten y superen su ansiedad. Los resultados reflejan la afectividad del programa diseñado.

\section{Palabras clave}

Aprendizaje, segunda lengua, autocontrol, ansiedad.

1 Carlos Alberto Armendáriz Valles. Profesor de la Universidad Pedagógica Nacional del Estado de Chihuahua, Campus Camargo, México. Es licenciado en relaciones industriales y maestro en educación básica. Correo electrónico: caarmendarizv@gmail.com

ID: http://orcid.org/0000-0001-9780-7676 
RECIE. Revista Electrónica Científica de Investigación Educativa

Vol. 4, núm. 2, enero-diciembre 2019, pp. 955-969.

\begin{abstract}
This intervention project was designed based on the need to help students to control the anxiety produced by second language learning, are intervened educationally in order to work strategies that allow them to be aware of the anxiety generated by the oral practices, written and auditory exercises in the English subject. It is also intended to develop self-control strategies, facilitating the learning and management of the language, as well as maneuver negative anxiety to become positive and propel second language learning. The diagnostic made reveals that the students recognize the anxiety and know that there is a defense mechanism due to the insecurity they have when learning a foreign language, this allows them to control their emotion, resulting in a better language learning. The strategies were worked with seven activities designed to create trust climate and recognition of the psychological processes that are being in a group and provide security, encouraging students to face and overcome their anxiety. The results reflect the effectiveness of the designed program.
\end{abstract}

\title{
Keywords
}

Learning, second language, self-control, anxiety.

\section{Introducción}

La necesidad de los seres humanos de comunicarse se remonta hasta la prehistoria, buscando la manera de expresarse, paulatinamente se fue estructurando un medio de comunicación que llego a ser el lenguaje, el cual tomo forma matizado con aspectos geográficos, históricos y culturales propios del contexto. En todas las épocas del mundo, ha sido necesario el conocimiento de otra lengua adicional a la materna para poder comunicarse entre los pueblos, los intereses culturales, políticos y económicos acrecentaron esta necesidad.

El aprendizaje de una segunda lengua produce ansiedad y es diferente de otros tipos de ansiedad general y existe una relación negativa entre dicha ansiedad y como se promueve el desarrollo de determinadas competencias en la segunda lengua. Algunos autores han descrito el mecanismo por el cual puede surgir la ansiedad en un estudiante en sus primeras etapas de aprendizaje, encontrando muchas dificultades, que pueden ir desde la incapacidad para producir la fonética de la lengua extranjera, problemas inherentes a la comprensión de la semántica y los modismos de cada región. 
Hoy en día, es necesario proveer a la sociedad con ciudadanos que tengan la capacidad de desarrollar su Inteligencia emocional y sus habilidades socioemocionales, el controlar la ansiedad es una de ellas, lo cual les será útil para un adecuado desempeño en su vida académica, especialmente en el aprendizaje de una segunda lengua, dos factores importantes propuestos en el Modelo educativo 2018.

El diagnostico, así como el desarrollo del proyecto de intervención se efectuó en la escuela primaria estatal Edmundo Porras Fierro No. 2212, ubicada en la Calle Comonfort s/n Cd. Camargo, Chihuahua. El grupo seleccionado fue el cuarto grado. ya que, conforme al diagnóstico, es el grupo en donde se localizaron mayores indicadores de ansiedad.

\section{Planteamiento del problema}

Durante la práctica docente en la enseñanza del idioma inglés, en los cuales se registraron memorias en diarios anecdóticos durante tres años, se detectó un patrón recurrente en todos los grupos, especialmente en el cuarto grado, correspondiente al ciclo escolar 2015-2016.

Con fundamento en los datos encontrados se formuló la siguiente pregunta, ¿Cómo superar la ansiedad que se genera en los alumnos del grupo de 4‥ Grado de la Escuela Primaria Edmundo Porras Fierro de Ciudad Camargo Chihuahua, al realizar las prácticas y ejercicios orales, escritos y auditivos de la asignatura de Ingles?

\section{Objetivo General}

- Diseño de estrategias educativas orientadas a favorecer el conocimiento y autocontrol en los alumnos de educación primaria de la ansiedad que produce el aprendizaje de una segunda lengua.

\section{Objetivo Específico}

- Trabajar en los alumnos estrategias que les permita ser conscientes de la ansiedad que les genera la práctica de ejercicios orales, escritos y auditivos.

\section{Metodología}

Para elaborar el diagnostico se utilizó el paradigma cualitativo, como método la investigación acción y como instrumentos de recolección de datos: observación participante, registro en diario de campo, entrevistas individuales y grupales, aplicación del auto reporte de ansiedad para niños y adolescentes utilizado en México en el sistema nacional de salud (Cuadro 1) y Foreign Language Classroom Anxiety Scale (FLCAS) Escala de Ansiedad en 
RECIE. Revista Electrónica Científica de Investigación Educativa Vol. 4, núm. 2, enero-diciembre 2019, pp. 955-969.

las Clases de Lengua Extranjera de la autora Hellen Horwits (1986) Cuadro 2. La investigación fue debidamente autorizada por la dirección de la escuela.

Con base en los síntomas detectados y registrados en diario de campo, al sistematizar la información dio como resultado las siguientes categorías:

a) Aspectos cognitivos displacenteros, alto grado de tensión emocional, irritabilidad, falta de concentración y sensación de mente en blanco.

b) Aspectos fisiológicos, síntomas respiratorios, gastrointestinales, y musculares, caracterizados por respiración agitada y dolores abdominales, sensación de ahogo o falta de aliento, opresión o malestar torácico, náuseas o mareo

c) Aspectos motores, hiperactividad, paralización motora, movimientos torpes o repetitivos, conductas de evitación, dificultad de expresión verbal que suelen implicar comportamientos poco racionales y escasamente adaptativos. Los alumnos presentaban preocupación excesiva, pensamientos negativos o de frustración y dificultad para concentrarse.

d) Huida/evitación: solicitar ausentarse del salón con regularidad. La aplicación del Auto reporte de ansiedad para niños y adolescentes (AAA) de la Clínica de Adolescentes del Instituto Nacional de Psiquiatría RFM, México, adaptada por el Dr. francisco de la Peña Olveda, dio como resultado que el total de los alumnos eligieron la opción "siempre" en al menos 10 de los 41 aspectos a evaluar, lo que es considerado como niño ansioso, según el Instituto.

En cuanto a la aplicación del Foreign Language Classroom Anxiety Scale (FLCAS) Escala de Ansiedad en las Clases de Lengua Extranjera de la autora de Hellen Horwits (1986), el total de los alumnos eligieron como mínimo 8 aspectos totalmente de acuerdo y 8 totalmente en desacuerdo (Ítems afirmativos de situación ansiosa) del total de 33 reactivos. Dando como resultado un alto grado de ansiedad en el aprendizaje de una segunda lengua.

La intervención se diseña basada en el programa cognitivo conductual de Philip C. Kendall (1990), dicho programa fue orientado para tratar trastornos de ansiedad por separación infantil TAS y trastorno de ansiedad generalizada TAG, tomando como referencia los principios del programa, se diseña un taller el cual incluye siete etapas, tres sesiones de cincuenta minutos cada una, programados de tal forma que en la primera etapa, los alumnos, Identifiquen la emoción de la ansiedad, en la segunda 
logren categorizar esa emoción, en una tercera etapa reconozcan la ansiedad como una emoción inherente a su propia naturaleza e identifique episodios pasados de crisis de ansiedad, en una cuarta etapa logren controlarla, en una quinta etapa alienten su motivación intrínseca a través de apoyo extrínseco, en la sexta etapa practicaran habilidades adquiridas y en la última etapa, reforzaran el control de la ansiedad aprendiendo ejercicios de relajación a través de yoga. El programa general de actividades se especifica en Cuadro 3.

\section{Referencias teóricos}

El presente proyecto de intervención se desarrolla desde dos ámbitos, la psicología social y los aportes psicopedagógicos en la enseñanza de una segunda lengua, se recurre a diversos autores que abordan el tema de la ansiedad general y la ansiedad por aprendizaje de una segunda lengua. En cuanto a psicología social se retoman las ideas Ricardo Pichón Riviere (1968) quien conceptualiza el tema como el miedo que se genera en las personas frente a todo intento de adaptación, indica que existen dos tipos de ansiedad básica: el miedo a la pérdida y el miedo al ataque, y frente a las cuales se instrumentaran medidas defensivas. La ansiedad aparece cuando emergen los primeros indicios del cambio, y más concretamente, en toda situación de aprendizaje, haciéndose extensiva a todo proceso de interacción, a todo tipo de manipuleo o apropiación de lo real, a todo intento de respuesta coherente y significativa de las demandas de la realidad (adaptación), lo cual se traduce en: a) miedo a la pérdida del equilibrio ya logrado en la situación anterior, y b) miedo al ataque de la nueva situación en la que el sujeto no se siente adecuadamente instrumentado. Ambos subsisten y cooperan, cuando su monto aumenta, configuran la ansiedad y resistencia ante el cambio. Una característica fundamental de la ansiedad es su monto o intensidad, lo que hará que ellas sean tolerables o intolerables. En relación a la ansiedad por aprendizaje de una segunda lengua, se hace referencia a Helen k. Horwitz (1986) quien ve la ansiedad como un complejo único de autopercepciones, creencias, sentimientos y comportamientos relacionados con el aprendizaje de un idioma y que se desprende de la especificidad inherente a dicho aprendizaje.

Para Horwitz (1986) esta ansiedad brota de tres fuentes principales: la aprensión comunicativa, el miedo a una evaluación negativa por parte de otros y la ansiedad por los exámenes.

Gardner y Macintyre (1991) lograron demostrar que la ansiedad ante el idioma es diferente de otros tipos de ansiedad general y que existe una correlación negativa entre dicha ansiedad y como se ejecutaban 
determinadas tareas en la segunda lengua. Los autores han descrito el mecanismo por el cual puede surgir la ansiedad, un estudiante en sus primeras etapas de aprendizaje, podría encontrarse con muchas dificultades, que podrían ir desde la incapacidad para producir los sonidos de la lengua materna hasta los problemas inherentes a la adquisición de la nueva gramática. Si estas primeras experiencias le ponen nervioso $\mathrm{y}$, además, el alumno se siente incómodo de cometer errores, se desarrolla la denominada ansiedad de situación, con la repetición de este fenómeno, que se va retroalimentando así mismo, se encuentra la génesis del problema.

\section{Resultados}

Los alumnos tienen dificultad para reconocer sus emociones, lo cual es normal por la etapa de desarrollo en la que se encuentran, sus edades oscilan entre los nueve y diez años, aun no llegan a la etapa de operaciones concretas.

La primera etapa de aplicación tuvo como objetivo la identificación de emociones que podemos llamar positivas y negativas, los niños reconocen con más facilidad las emociones positivas relacionadas con aspectos placenteros y se turban ante los que no pueden reconocer o identificar. Conforme fueron explorando estas emociones, fueron conscientes de que había algo que les causaba molestia y les impedía desempeñarse adecuadamente en el salón de clases.

En la implementación de las actividades de la segunda etapa, una vez identificado las emociones y sentimientos negativos, fueron capaces con facilidad darles una valoración a estos en una escala de menos a más perturbadores. Es fácil para el infante darle un valor más que identificarlo.

La tercera actividad permitió que los niños lograron interiorizar y por lo tanto ser conscientes de sus reacciones anteriores y ser constantes en las nuevas formas de pensamientos que lograron, de la misma forma, escucharon grabaciones de sí mismos ejecutando lecturas de ejercicios en los cuales mostraban signos de ansiedad, lo cual les permitió hacer una comparación entre sus distintas reacciones.

Los niños fueron madurando sus sentimientos y por lo tanto sus reacciones ante lo desconocido, identificando lo que les causa inquietud o molestia al leer, hablar o escuchar ejercicios en una lengua extranjera.

La analogía que se hizo en la cuarta etapa, de un monstruo que representaba ese recién identificada emoción, luchando contra otro personaje que los representaba a si mismos, fue sumamente enriquecedor 
para ellos. Se utilizó una actividad lúdica para vencer emociones y sentimientos reales.

En la actuación de roles, el niño identifico, categorizo y fue consciente de sus reacciones y la de sus compañeros, al interpretar roles que caracterizaban las conductas de sus compañeros, logrando con esto que hicieran una autocrítica al actuar estos roles.

En la última de las etapas, se alcanzó el reforzamiento de las competencias adquiridas, es científicamente conocido que el ejercicio relajo y por lo tanto disminuye la ansiedad. A los niños les agrado hacer ejercicio, los cuales se complementaron con técnicas de respiración utilizando métodos de yoga.

\section{Conclusiones}

Existe una dificultad de los niños para reconocer la ansiedad como parte de su capacidad adaptativa, pero llegan a comprenderla con el asesoramiento y las técnicas adecuadas.

El desarrollo y gravedad de la misma depende de múltiples factores como: herencia genética, conductas aprendidas de los padres o figuras de apego, ambiente familiar y experiencias vividas, lo cual afecta directamente en el desempeño escolar.

La ansiedad en la escuela se desarrolla inicialmente cuando el niño que ingresa a primer grado siente un miedo a la pérdida o ansiedad por separación, un segundo factor que influye determinantemente es la sociabilización con maestros y compañeros de clase, si esta experiencia no ha sido grata, desarrollara ansiedad.

En el aprendizaje de una segunda lengua se caracterizan síntomas específicos ya que el alumno tiene que desarrollar competencias oral, escrita y auditiva, derivado de enfrentar una estructura gramatical (sintaxis, semántica y modismos), que es completamente distinta a su idioma natal, además de la obligación de aprender pronunciación y entonación.

El temor a la burla de los compañeros tiene una relevancia mayor en el alumno que la presencia del maestro, ya que comprende que el docente le ayudara en el caso de que se equivoque, aun y cuando este puede ser intimidante por la figura de autoridad.

Atendiendo al contenido temático que indica el programa nacional de ingles, se encontró que los temas que requieren de conocimientos en semántica y fonética son los que producen mayor ansiedad. Utilizando estrategias de enseñanza aprendizaje lúdicas, disminuye este factor, aunado a esto, existe una barrera cultural de oposición a aprender una segunda lengua. 
Un aspecto relevante fue que los niños que no alcanzaron los objetivos que se propusieron para el desarrollo del presente trabajo o que lo lograron medianamente, provienen de hogares disfuncionales, en donde la atención de uno de los padres es deficiente o su primera figura de apego no son los padres.

Los niños con un grado de ansiedad elevada, los provenientes de un hogar disfuncional o que presentan agresividad, tuvieron dificultad para reconocer sus propias cualidades, es más fácil para ellos reconocer los aspectos negativos de si mismos, de los cuales, en ocasiones se sienten orgullosos. Cuando a estos niños se les solicita realicen un dibujo y escriban aspectos negativos de su comportamiento, escriben estos aspectos en el lado izquierdo de la hoja. Otra situación muy notoria, es que tienden a colocarse en lugares alejados de la puerta y del maestro para trabajar, normalmente al lado izquierdo del salón. Los trazos que realizan son gruesos y con tallones.

A los niños les agrada reflejarse en videograbaciones, aprenden observando su propia actuación y no sienten temor de expresar una autocrítica.

Con base en los hallazgos se sugiere incluir dentro de la práctica docente, en el desarrollo de la carpeta evolutiva, capítulos para el desarrollo Psicológico-emocional de nuestros alumnos, a la vez de que nos proporcionaría elementos para otorgar aprendizajes significativos, nos ayudaría a desarrollar inteligencia emocional y habilidades socioemocionales, las cuales son uno de los apartados del Modelo Educativo 2018.

\section{Referencias}

Abello, G:C. (2008). Investigacion educativa o investigacion pedagogica? Revista Internacional de Investigacion yEducacion, Vol 1, num 1, juliodiciembre 2008. pp 163-173.

Alvarez, N. (04- 03-2013).

http://comunicacioneyc.blogspot.com/2013/03/lenguaje-teoria-

piaget-aprendido-cuando.html. Obtenido de Comunicacion, Educacion y Cultura.

Arduin, J., Bustos, C., \& Jarpa, M. (1998). La Adquisicion del Lenguaje en los Niños. Obtenido

de http://www.robertexto.com/archivo17/lenguaje_ninios.htm

Beyer, R. (23- 04 - 2015). Pichon-riviere y la teoria del aprendizaje. Obtenido de Pichon-Riviere y la Teoria del Aprendizaje: 
http://milnovecientossesentayocho.blogspot.mx/2015/04/pichonriviere-y-la-teoria-del.html

Bleger, J. (23-04-2015). El aprendizaje. 1968/Grupalista biblioteca de Psicologia Social Pichoniana. Obtenido de http://milnovecientossesentayocho.blogspot.mx/2015/04/pichonriviere-y-la-teoria-del.html

Horwitz, E. (1983). “F.L.C.A.S.” Unpubished Manuscript. Austin: University of Texas.

Horwitz, E. K. Horwitz, M. B. \& Cope, J. A. (1991) "Foreing Languaje Clasroom Anxiety" Languaje Anxiety: From Theory and Resaerch to Classrooms Implications. Ed. Horwitz, Elaine K. \& Young, Dolly J. Englewood Cliffs, NJ: Pretentice Hall.

Macintyre, P. D. \& Gardner, R.C.( 1994) "The Subtle Effects of Languaje Anxity on Cognitive Processing in Second Languaje". Language Learning 44(2): 283-305.

Rivera, L. (2011). Terapia De Juego Para La Ansiedad. Salud Emocional, 26. Obtenido de http://clinicandpsique.blogspot.mx/2011/08/ninoscon-atencion-dispersa-que-es-y.html

Philip, C. K. (2010). Terapia Cognitivo-Conductual Para trastornos de ansiedad en niños. Buenos Aires, Argentina; Librería Acadia.

UPN. (1998). Elementos Basicos de Investigacion Cualitativa. Morelos, Mexico: UPN. Obtenido de http://upnmorelos.edu.mx/assets/elementos-basicos-deinestigacion-cualitativa.pdf

Vigosky, L.S. (1988). "El desarrollo de los procesos psicológicos superiores" México D.F. Grijalbo

\section{Tablas y cuadros}

\section{Cuadro 1}

Auto reporte de ansiedad para niños y adolescentes (AAA) Clínica de Adolescentes del Instituto Nacional de Psiquiatría RFM, México Adaptada por el Dr. francisco de la Peña Olveda, adolesc@imp.edu.mx Nombre Edad Sexo

Fecha Expediente

Instrucciones: Por favor Lee con cuidado cada una de las oraciones y marca con una $\mathrm{x}$ que tanto se aplica a tu caso, para nunca, algunas veces o siempre en las dos últimas semanas. No hay respuestas buenas ni malas. 
RECIE. Revista Electrónica Científica de Investigación Educativa Vol. 4, núm. 2, enero-diciembre 2019, pp. 955-969.

\begin{tabular}{|c|c|c|c|c|}
\hline 1 & Cuando tengo miedo no respiro bien. & Nunca & $\begin{array}{l}\text { Algunas } \\
\text { veces }\end{array}$ & siempre \\
\hline 2 & $\begin{array}{l}\text { Cuando estoy en la escuela me duele } \\
\text { la cabeza. }\end{array}$ & & & \\
\hline 3 & $\begin{array}{l}\text { Me molesta estar con personas que no } \\
\text { conozco. }\end{array}$ & & & \\
\hline 4 & $\begin{array}{l}\text { Cuando duermo en una casa que no es } \\
\text { la mía siento miedo. }\end{array}$ & & & \\
\hline 5 & $\begin{array}{l}\text { Me preocupa saber si le caigo bien a } \\
\text { las otras personas. }\end{array}$ & & & \\
\hline 6 & $\begin{array}{l}\text { Cuando tengo miedo, siento que me } \\
\text { voy a desmayar. }\end{array}$ & & & \\
\hline 7 & Soy nervioso (a). & & & \\
\hline 8 & Sigo a mis papas a donde ellos van. & & & \\
\hline 9 & $\begin{array}{l}\text { Las personas me dicen que me veo } \\
\text { nervioso (a). }\end{array}$ & & & \\
\hline 10 & $\begin{array}{l}\text { Me pongo nervioso (a) cuando estoy } \\
\text { con una persona que no conozco. }\end{array}$ & & & \\
\hline 11 & $\begin{array}{l}\text { Cuando estoy en la escuela me duele } \\
\text { la panza. }\end{array}$ & & & \\
\hline 12 & $\begin{array}{l}\text { Cuando siento mucho miedo, siento } \\
\text { que me voy a volver loco (a). }\end{array}$ & & & \\
\hline 13 & $\begin{array}{l}\text { Me siento preocupado cuando } \\
\text { duermo solo (a). }\end{array}$ & & & \\
\hline 14 & $\begin{array}{l}\text { Me preocupo de ser tan bueno (a) } \\
\text { como los otros niños, por ejemplo en } \\
\text { la escuela o deportes. }\end{array}$ & & & \\
\hline 15 & $\begin{array}{l}\text { Cuando tengo mucho miedo, siento } \\
\text { como si las cosas no fueran reales. }\end{array}$ & & & \\
\hline 16 & $\begin{array}{l}\text { En las noches sueño que cosas feas le } \\
\text { van a pasar a mis papas. }\end{array}$ & & & \\
\hline 17 & $\begin{array}{l}\text { Me preocupo cuando tengo que ir a la } \\
\text { escuela. }\end{array}$ & & & \\
\hline 18 & $\begin{array}{l}\text { Cuando tengo mucho miedo, } \mathrm{mi} \\
\text { corazón late muy rápido. }\end{array}$ & & & \\
\hline 19 & Tiemblo. & & & \\
\hline 20 & En las noches siento que me va a & & & \\
\hline
\end{tabular}




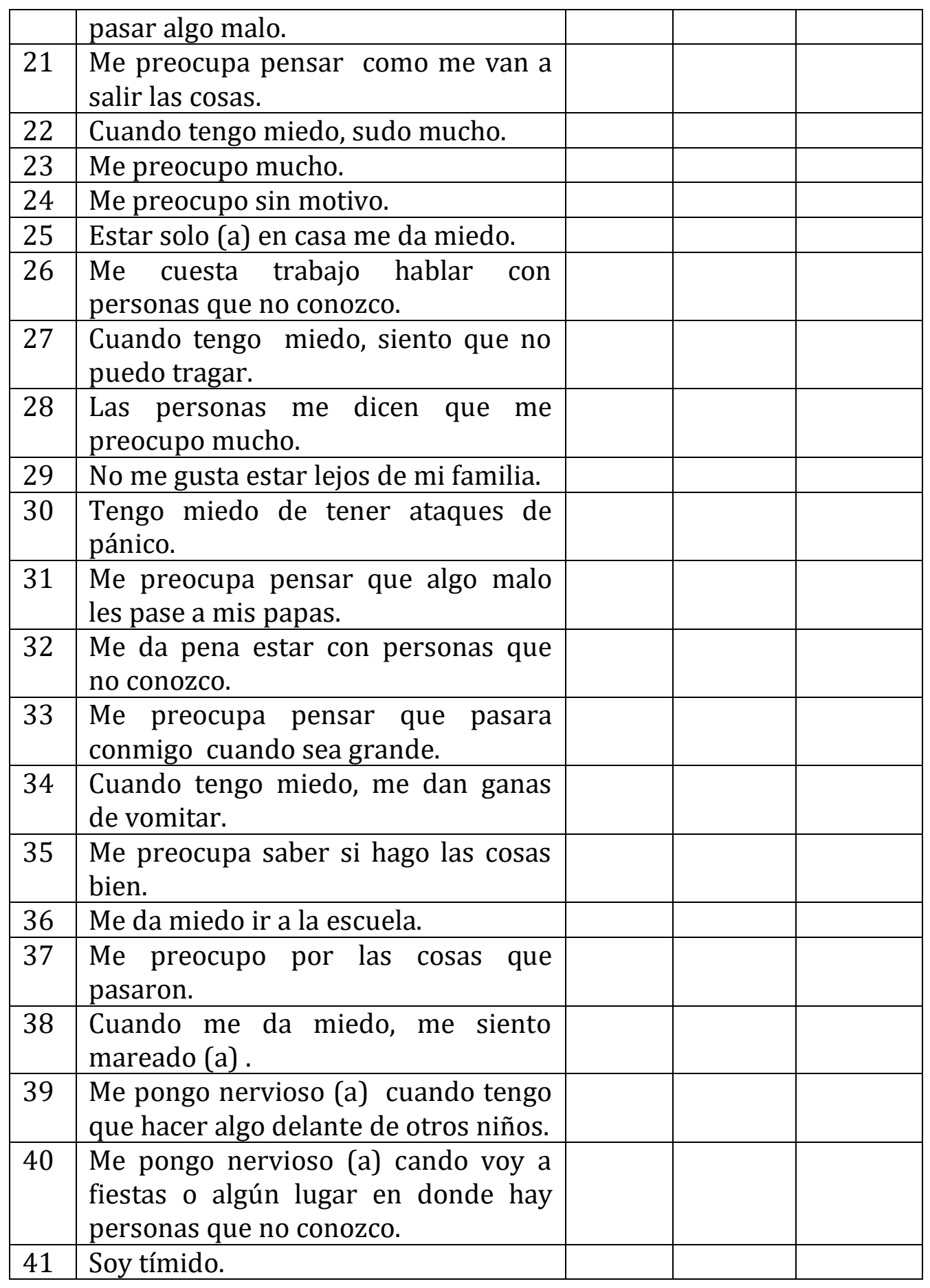

Superando la ansiedad para lograr el aprendizaje de una segunda lengua... 
RECIE. Revista Electrónica Científica de Investigación Educativa Vol. 4, núm. 2, enero-diciembre 2019, pp. 955-969.

Guía clínica para los trastornos de ansiedad en niños y adolescentes. Instituto nacional de psiquiatría Ramón de la Fuente Muñiz. México D. F. 2010. Eva Marcela

Cárdenas, Miriam Feria, Lino Palacios, Francisco de la Peña. Secretaria de Salud. La escala de evaluación SCARED por sus siglas en inglés ( Screen For Child Anxiety Related Emotional Disorders), Auto reporte de ansiedad para niños y adolescentes

(AAA) en español, fue diseñado como una herramienta de tamizaje de los trastornos de ansiedad en los niños. Consta de 41 reactivos, los cuales se clasifican de 0 a 2, siendo 0 nunca, 1 algunas veces y 2 siempre. El punto de corte es 25 , es decir, existe una probabilidad del $70 \%$ de que el niño o adolescente padezca algún trastorno de ansiedad.

\section{Cuadro 2}

Foreign Language Classroom Anxiety Scale

FLCAS Escala de Ansiedad en las Clases de Lengua Extranjera

\section{Instrucciones:}

Las siguientes afirmaciones se refieren a diversas situaciones frecuentes en el aprendizaje de un idioma. Su tarea consiste en valorar su grado de acuerdo o desacuerdo con cada una de las siguientes afirmaciones, respecto a sus clases de inglés.

\begin{tabular}{|c|c|c|c|c|}
\hline $\begin{array}{c}\text { Totalmente } \\
\text { de } \\
\text { Acuerdo } \\
1\end{array}$ & De acuerdo & No sé & En desacuerdo & $\begin{array}{c}\text { Totalmente } \\
\text { en desacuerdo } \\
5\end{array}$ \\
\hline
\end{tabular}

\begin{tabular}{|l|l|l|l|l|l|}
\hline \multicolumn{1}{|c|}{ Aspecto a evaluar } & 1 & 2 & 3 & 4 & 5 \\
\hline $\begin{array}{l}\text { Nunca estoy completamente seguro/a de mí mismo } \\
\text { cuando hablo en la clase. }\end{array}$ & & & & & \\
\hline No me preocupa cometer errores en la clase & & & & & \\
\hline Tiemblo cuando sé que me van a preguntar en clase. & & & & & \\
\hline $\begin{array}{l}\text { Me asusta no entender lo que el profesor/a está } \\
\text { diciendo en } \\
\text { Inglés. }\end{array}$ & & & & & \\
\hline $\begin{array}{l}\text { No me molestaría en absoluto asistir a más clases de } \\
\text { inglés }\end{array}$ & & & & & \\
\hline $\begin{array}{l}\text { Durante las clases, pienso en cosas que no tienen nada } \\
\text { que ver con la clase. }\end{array}$ & & & & & \\
\hline
\end{tabular}




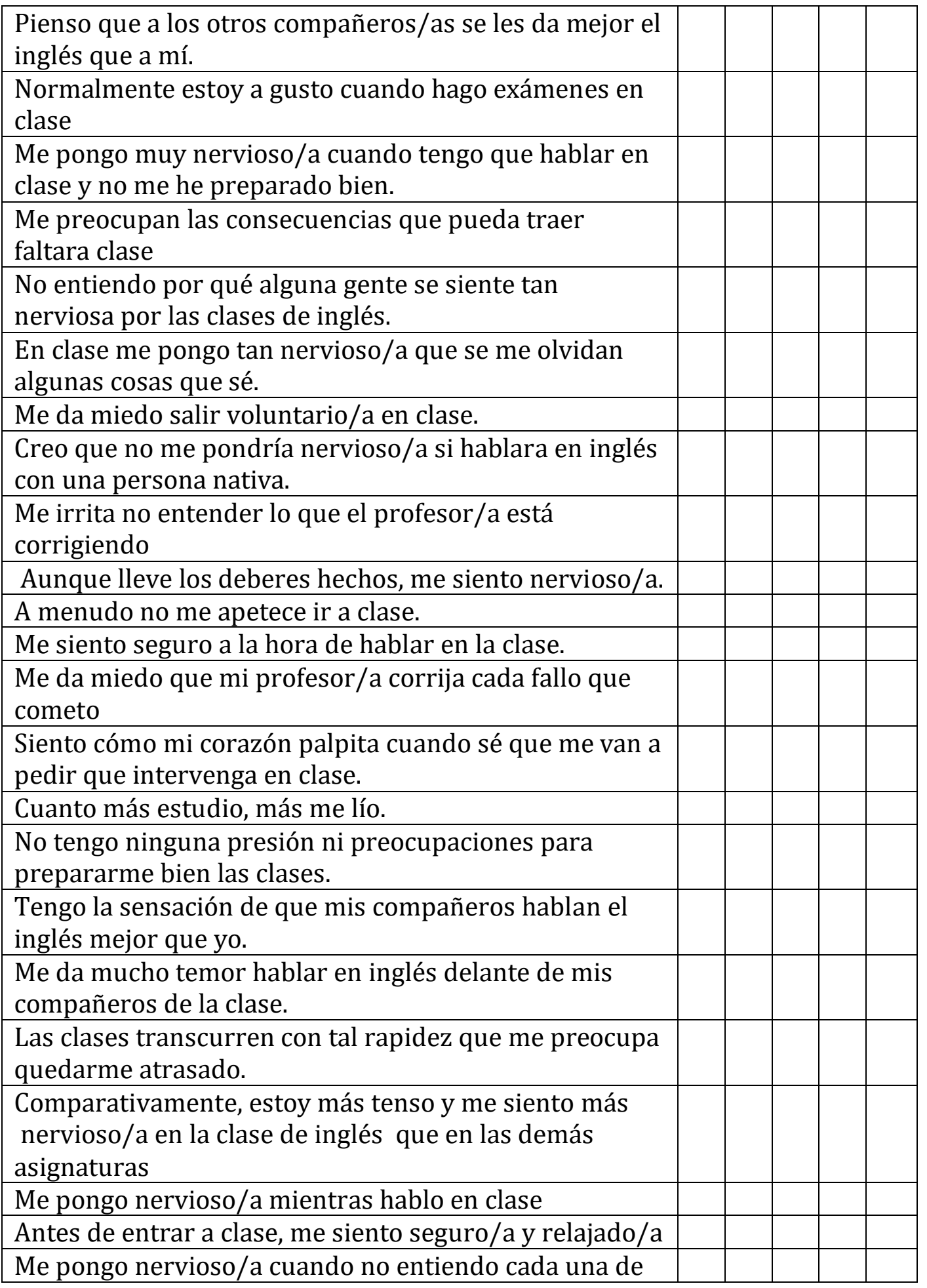

Superando la ansiedad para lograr el aprendizaje de una segunda lengua... 
RECIE. Revista Electrónica Científica de Investigación Educativa Vol. 4, núm. 2, enero-diciembre 2019, pp. 955-969.

\begin{tabular}{|l|l|l|l|l|}
\hline la palabra que mi profesor/a dice. & & & & \\
\hline $\begin{array}{l}\text { Me abruma la cantidad de cosas que hay que aprender } \\
\text { para poder hablar inglés. }\end{array}$ & & & & \\
\hline $\begin{array}{l}\text { Temo que mis compañeros/as de clase de inglés de rían } \\
\text { de mí cuando hablo en inglés. }\end{array}$ & & & \\
\hline $\begin{array}{l}\text { Creo que me sentiría a gusto hablando entre nativos/as } \\
\text { que } \\
\text { hablan inglés. }\end{array}$ & & & & \\
\hline $\begin{array}{l}\text { Me pongo nervioso/a cuando el profesor/a pregunta } \\
\text { cosas que no he podido preparar. }\end{array}$ & & & & \\
\hline
\end{tabular}

Foreign Language Classroom Anxiety. Elaine K. Horwitz, Michael B. Horwitz, Joann

Cope. The Modern Language Journal. Vol. 70, No. 2 .Pag. 308-309.

https://www.jstor.org/stable/327317?seq=1\#page_scan_tab_contents

Traducción. Carlos Alberto Armendáriz Valles.

La presente escala mide variables que producen ansiedad en el aprendizaje de una segunda lengua, variables aisladas de la ansiedad y variables de confirmación. La escala fluctúa del uno al cinco, en donde uno tiene una referencia de totalmente de acuerdo y cinco referencias de totalmente en desacuerdo.

\section{Cuadro 3}

Organización general de actividades.

\begin{tabular}{|c|c|c|c|c|}
\hline Etapa del & Etapa Cognitiva & \multicolumn{2}{|l|}{ Competencia } & Actividad \\
\hline $\begin{array}{l}\text { Dibujando } \\
\text { mi retrato }\end{array}$ & $\begin{array}{l}\text { Identificación de } \\
\text { emociones y } \\
\text { sentimientos }\end{array}$ & $\begin{array}{l}\text { Reconoce } \\
\text { sus } \\
\text { emociones } \\
\text { y } \\
\text { sentimiento } \\
\text { s y el } \\
\text { estímulo } \\
\text { que las } \\
\text { origina. }\end{array}$ & $\begin{array}{l}\text { a) } \\
\text { Conocerse } \\
\text { a sí mismo. } \\
\text { b) } \\
\text { Identifica } \\
\text { emociones } \\
\text { y } \\
\text { sentimiento } \\
\text { s negativos. } \\
\text { c) Valora } \\
\text { sus } \\
\text { cualidades }\end{array}$ & $\begin{array}{l}\text {-Dibujo de } \\
\text { silueta. } \\
\text {-Realiza una } \\
\text { Mascara. } \\
\text {-Autorretrato }\end{array}$ \\
\hline $\begin{array}{l}\text { Escalando } \\
\text { la montaña }\end{array}$ & $\begin{array}{l}\text { Jerarquización } \\
\text { de emociones y }\end{array}$ & \multicolumn{2}{|c|}{$\begin{array}{l}\text { Otorga un Valor a las } \\
\text { emociones en una escala. }\end{array}$} & $\begin{array}{l}\text { Escala en } \\
\text { forma de }\end{array}$ \\
\hline
\end{tabular}




\begin{tabular}{|c|c|c|c|}
\hline $\begin{array}{l}\text { del } \\
\text { monstruo } \\
\text { yeti. }\end{array}$ & $\begin{array}{l}\text { sentimientos } \\
\text { negativos. }\end{array}$ & & montaña. \\
\hline $\begin{array}{l}\text { Escuchando } \\
\text { mi propia } \\
\text { voz }\end{array}$ & Concientización. & $\begin{array}{l}\text { Analiza sus episodios de } \\
\text { ansiedad presentados con } \\
\text { anterioridad. }\end{array}$ & $\begin{array}{l}\text { Relato en el } \\
\text { cuaderno. }\end{array}$ \\
\hline $\begin{array}{l}\text { Enfrentand } \\
\text { o al Yeti. }\end{array}$ & Enfrentamiento. & $\begin{array}{l}\text { Enfrenta emociones para } \\
\text { ser consciente de ellas. }\end{array}$ & Comic. \\
\hline $\begin{array}{l}\text { Cartelolandi } \\
\text { a } \\
\text { "la tierra } \\
\text { de los } \\
\text { carteles." }\end{array}$ & $\begin{array}{l}\text { Motivación e } \\
\text { impulso. }\end{array}$ & $\begin{array}{l}\text { Desarrolla su motivación } \\
\text { intrínseca con apoyo de } \\
\text { motivación extrínseca de } \\
\text { sus compañeros. }\end{array}$ & $\begin{array}{l}\text { Carteles } \\
\text { motivacional } \\
\text { es }\end{array}$ \\
\hline $\begin{array}{l}\text { Actuación } \\
\text { de juego de } \\
\text { roles. }\end{array}$ & $\begin{array}{l}\text { Práctica de } \\
\text { habilidades } \\
\text { adquiridas. }\end{array}$ & $\begin{array}{l}\text { Representa diferentes } \\
\text { roles referentes a } \\
\text { episodios de ansiedad }\end{array}$ & Socio drama \\
\hline $\begin{array}{l}\text { Jugando al } \\
\text { yoga. }\end{array}$ & Reforzamiento. & $\begin{array}{l}\text { Practica ejercicios de } \\
\text { relajación y respiración a } \\
\text { forma de prevención para } \\
\text { evitar nuevos episodios } \\
\text { de ansiedad. }\end{array}$ & $\begin{array}{l}\text { 1.-Rutina de } \\
\text { ejercicios de } \\
\text { yoga } \\
\text { 2.-Platica a } \\
\text { alumnos de } \\
\text { otros grados. }\end{array}$ \\
\hline
\end{tabular}

El presente cuadro muestra el diseño de actividades del taller implementado en la intervención educativa, las actividades se encuentran secuenciadas en orden cronológico de aplicación, cada una de las siete etapas del taller fue diseñada conforme a una etapa cognitiva conductual. 
RECIE. Revista Electrónica Científica de Investigación Educativa Vol. 4, núm. 2, enero-diciembre 2019, pp. 955-969. 